\title{
QUANTIFICATION OF INTER-TRIAL NON-STATIONARITY IN SPIKE TRAINS FROM PERIODICALLY STIMULATED NEURAL CULTURES
}

\author{
Il “Memming” Park and José C. Príncipe \\ University of Florida \\ Department of Biomedical Engineering \\ Department of Electrical and Computer Engineering \\ Gainesville, FL 32611
}

\begin{abstract}
In neuroscience, non-stationarity detection of spike trains is useful for ensuring stability of experimental condition, and detecting plasticity. A novel method for estimating point process divergence and its application for non-stationarity detection in spike trains is proposed. The method for measuring divergence is based on decomposition of finite point process and Hilbertian metrics. The method is demonstrated by detecting short-term and long-term plasticity in neural culture probed with periodic stimulations.
\end{abstract}

Index Terms - non-stationarity detection, point process divergence, finite point process

\section{INTRODUCTION}

Independence and stationarity across trials are essential requirements for the analysis of experimental observations. Testing whether the measured dataset has the desired property is a challenging statistical problem, even more so when the measurements are in the form of spike trains. The best we can do is to use sensitive methods to detect non-stationarity and support the claim of stationarity from failure of detection. We introduce a novel method for point process divergence, and its application for non-stationarity detection in spike trains from periodically probed neural system. The point process divergence can be used to perform hypothesis testing for the inter-trial non-stationarity. As an application, we apply a collection of methods to claim the short-term non-stationarity and stationarity of datasets collected from neural cultures.

\section{NON-STATIONARITY OF SPIKE TRAINS FROM PERIODICALLY STIMULATED NEURAL CULTURE}

Periodically repeated stimulation to neural system is a standard technique to probe and analyze the state of the system. Certainly this is not a natural input for in vivo neural systems where the stimulus is more or less random, but the resulting spike train signals are easier to analyze; in many cases the response patterns are strictly time locked to the stimulation. From the dataset observed from neural cultures, we have previously observed that trial-to-trial variability plays a major role for classification or decoding of the repeated input stimulus conditions [1].

For a single probing trial, the measured spike train is not stationary in general. However, multiple trials can be considered independent and stationary through proper initialization, for example by controlling the inter-trial interval. By inter-trial stationarity, we mean that the probability law behind the set of trials do not change over time. In other words, if a set of trials obey inter-trial stationarity, each trial is independent and identically distributed. When the observations are spike trains, this means that the trials are independent realizations of the same point process (non-stationary within the trial in general). Given a measure of divergence between two sets of spike trains, we can perform statistical test to show if the underlying point processes are different, hence, showing inter-trial non-stationarity.

When electrically stimulated, the response of neural culture recorded through a micro-electrode array (MEA) changes as the stimulation is periodically repeated. Various resources of neurons are used and short term plasticity is first observed from the action potential timings. However, after a while the responses stabilize due to homeostatic mechanisms. The time period that this happens is related to the time constants of the neurophysiological events. Hence, when stimulated for a long time, the response becomes more or less stationary. In neural cultures, most action potentials occur time locked to the stimulation time, therefore this is visually verifiable. Assuming a certain portion of the trials to be inter-trial stationary, we would like to determine by how much we can extend the assumption.

\section{POINT PROCESS DIVERGENCE BASED ON FINITE POINT PROCESS MODEL}

Given two sets of spike trains, we would like to test whether they originate from the same probability law or not. A wide variety of statistics can be used for this purpose including mean firing rate, inter spike interval distribution, Fano factor, time to first spike distribution, and so on. However, none of these statistic listed above is universal in the sense that given a large number of spike trains from two distinct arbitrary point processes, the test guarantees it will distinguish them, because these summarizing statistics only capture partial characteristics of the underlying process. We need to compute a divergence statistic that originates from a full description of point processes. The finite point process is suitable for stimulus time locked observations where temporal coding is often observed and expected, since several trials of rapidly changing observations are available.

By definition, any realization of a finite point process has finite events with probability 1 . For a fixed bounded time interval, the total number of action potentials that can occur is limited by biophysical constraints such as refractory mechanisms, hence it is a finite point process. In this case, it is natural to decompose the statistic into the count statistic which describes the total number of action potentials, and the location statistic which describes the joint distribution of exact timing of action potentials given the total number [2]. The de- 
composition allows nonparametric estimation of any finite point process, effective especially when the total number is relatively small, which can be achieved by choosing a smaller interval for analysis.

Once we have a probability measure that describes the dataset, we can use a divergence on probability measures as a statistic to perform hypothesis testing. Several such divergences have been proposed (e.g. $[3,4,5,6])$. The proposed nonparametric estimation can be directly plugged into most of them.

Of most interest is the family of divergences that corresponds to a distance in a Hilbert space, known as Hilbertian metrics defined on probability measures [5]. Hein and Bousquet proposed a family of such metric for probability measures which can be embedded isometrically in a Hilbert space, hence suggesting a family of reproducing kernel Hilbert space (RKHS) for the probability measures. This family of Hilbertian metrics provides a foundation for designing kernels from generative models with non-Euclidean representation. We will explore the Hilbertian metrics applied to point processes. Note that our approach is applied to sets of spike trains unlike other spike train metrics that are defined on a pair of spike trains (e.g. [7, 8]).

Proposition 1 ([5]). Let $\mu_{1}$ and $\mu_{2}$ be two probability measures on $\mathcal{X}$, and $\mu$ be a measure that dominates both probability measures. Let $d_{\mathbb{R}^{+}}$be a $\frac{1}{2}$-homogeneous Hilbertian metric on $\mathbb{R}^{+}$. Then $d_{\mathcal{M}}$ defined as,

$$
d_{\mathcal{M}}^{2}\left(\mu_{1}, \mu_{2}\right)=\int_{\mathcal{X}} d_{\mathbb{R}^{+}}^{2}\left(\frac{\mathrm{d} \mu_{1}}{\mathrm{~d} \mu}, \frac{\mathrm{d} \mu_{2}}{\mathrm{~d} \mu}\right) \mathrm{d} \mu
$$

is a Hilbertian metric on $\mathcal{M} . d_{\mathcal{M}}^{2}$ is independent of the dominating measure $\mu$.

Let $\Omega$ be the set of all possible spike trains, and let $\Omega_{0}, \Omega_{1}, \cdots$ denote the partition of $\Omega$ such that $\Omega_{n}$ contains all possible spike trains with exactly $n$ action potentials within the time interval of interest. The probability measure $P$ for point process can also be decomposed according to the partition of $\Omega$. Define $P_{n}(A)=P(A \cap$ $\left.\Omega_{n}\right)$, then we can write $P=\sum_{n=0}^{\infty} P_{n}$. Also denote $P_{n}(\Omega)$ as $p_{n}$, the probability of having $n$ action potentials. $P$ is assumed to be absolutely continuous with respect to $\mu$ (extended Lebesgue measure of $\Omega$ ), thus we can take the Radon-Nikodym derivative,

$$
\begin{aligned}
\frac{\mathrm{d} P}{\mathrm{~d} \mu}(\omega) & =P_{0}(\Omega) \delta_{\Omega_{0}}(\omega)+\sum_{n=1}^{\infty} \frac{\mathrm{d} P_{n}}{\mathrm{~d} \mu}(\omega) \\
& =p_{0} \delta_{\Omega_{0}}(\omega)+\sum_{n=1}^{\infty} p_{n} f_{n}(\omega)
\end{aligned}
$$

where $f_{n}$ is the unordered joint location density; $f_{n}$ is symmetric on the permutation of its arguments. $f_{n}$ has a close relationship with the density of Janossy measure $j_{n}(\omega)=n ! p_{n} f_{n}(\omega)$ [2].

Given a sequence of observations $X=\left\{x_{i}\right\}_{i=1}^{M}$, we can use the decomposition (3) for estimation of the finite point process. Let the subsequences $X^{(n)}=\left\{x_{i} ; x_{i} \in \Omega_{n}, i=1, \ldots, M\right\}$ be the set of all spike trains with exactly $n$ spikes. Frequency based estimate of the total count distribution and the kernel density estimation of $f_{n}$ can be written as,

$$
\begin{aligned}
\hat{p}_{n} & =\frac{\left|X^{(n)}\right|}{|X|} \quad n=0,1, \ldots \\
\hat{f}_{n}(\vec{t}) & =\frac{1}{\left|X^{(n)}\right|} \sum_{\vec{x} \in X^{(n)}} \kappa_{n}\left(\vec{x}-\vec{t} ; \sigma_{n}\right) \\
n & =1, \ldots
\end{aligned}
$$

where $\kappa_{n}(\cdot ; \sigma)$ is a symmetric $n$-dimensional density estimation kernel with bandwidth parameter $\sigma$ which is also symmetric on the permutation of its arguments. $\left\{\hat{p}_{n}, \hat{f}_{n}\right\}$ completely describes a general finite point process as we discussed before. In this paper, we used a spherical normal distribution with $\sigma_{n}=\left|X^{(n)}\right|^{-\frac{1}{5}} \cdot \sqrt{n} \cdot \sigma_{1}$ where $n \neq 1$ is the dimension and $\sigma_{1}$ is the bandwidth for a single sample in one dimension.

Plugging in $d_{\frac{1}{2} \mid 1}^{2}=(\sqrt{x}-\sqrt{y})^{2}$ (see [5]) for $d_{\mathbb{R}^{+}}^{2}$, and using the nonparametric estimator of finite point process (5) to (1) with respect to the reference measure $\mu$, we obtain the Hellinger distance estimator:

$d_{\mathcal{M}}^{2}(\hat{P}, \hat{Q})=\int_{\Omega} d_{\frac{1}{2} \mid 1}^{2}\left(\frac{\mathrm{d} \hat{P}}{\mathrm{~d} \mu}, \frac{\mathrm{d} \hat{Q}}{\mathrm{~d} \mu}\right) \mathrm{d} \mu=\int_{\Omega}\left(\sqrt{\frac{\mathrm{d} \hat{P}}{\mathrm{~d} \mu}}-\sqrt{\frac{\mathrm{d} \hat{Q}}{\mathrm{~d} \mu}}\right)^{2} \mathrm{~d} \mu$

where $\vec{t}=\left(t_{1}, \cdots, t_{n}\right)$, and $\mu$ is the extended Lebesgue measure.

Since the integration of (6) is not easily computable, we resort to Monte Carlo method for estimation. We would like to use the average probability measure $\nu=\frac{\hat{P}+\hat{Q}}{2}$ for importance sampling, however, $\nu$ is usually not absolutely continuous with respect to $\mu$. After some measure theoretic manipulation we obtain the following.

$$
d_{\mathcal{M}}^{2}(\hat{P}, \hat{Q})=\int_{\mathcal{X}} d_{\mathbb{R}^{+}}^{2}\left(\frac{\mathrm{d} \hat{P}}{\mathrm{~d} \mu}, \frac{\mathrm{d} \hat{Q}}{\mathrm{~d} \mu}\right) \frac{2}{\frac{\mathrm{d} \hat{P}}{\mathrm{~d} \mu}+\frac{\mathrm{d} \hat{Q}}{\mathrm{~d} \mu}} \mathrm{d} \nu
$$

In (7), we can either use all the original spike trains that was used to estimate $\hat{P}$ and $\hat{Q}$, or generate arbitrary number of spike trains from $\hat{P}$ and $\hat{Q}$. The latter approach provides more controlled variance but is slower. We used the former method in this paper.

\section{ARTIFICIAL DATA SETS}

In table 1, the proposed method is tested on 4 distinct point processes that have emphasis on features related to neuroscience; correlated spike timings [9], firing rate change [10], precisely timed spike trains [11], and serial correlation [12].

Experiment I is correlated versus independent precisely timed spike train model where the two conditions have identical marginal intensity functions. Two distinct spike train sets with at most two action potentials are constructed; common $t_{1} \sim \mathcal{U}(0.2,0.3)$ but $t_{2} \sim$ $t_{1}+0.3+\mathcal{N}(0,0.01)$ in one, and $t_{2} \sim \mathcal{U}(0.5,0.6)+\mathcal{N}(0,0.01)$ in the other. Both $t_{1}$ and $t_{2}$ have 0.1 probability of loss. Experiment II is a homogeneous Poisson model with different rates; 100 ms long with average 3 and 5 action potentials (we omit the details due to space, contact authors for details and code). Note that the proposed method is sensitive to all datasets while no other method is. Non-parametric method is a generalist, while each of the parametric method is a specialist. As expected, one can see from the table that the proposed method is a generalist.

\section{REAL DATA SETS}

The data sets are obtained from rat cortical culture grown on a 60 channel MEA. Briefly, embryonic day 18 Sprague/Dawley rat cortical hemispheres were dissociated and grown on an 8 by 8 grid of 30 $\mu m$ electrodes spaced $200 \mu m$ apart. Spiking activity was recorded at a sampling rate of $25 \mathrm{kHz}$ and action potentials were detected by thresholding five times standard deviation of the noise floor (for details see [1]). Each culture was electrically stimulated at $20 \mathrm{~Hz}$ on 


\begin{tabular}{c|c|c|c|c|c|c|c|c|c} 
Experiment & FR & FF & ISI & N & $L_{2} 100$ & $L_{2} 10$ & TTFS & HL100 & HL10 \\
\hline I & 0.0685 & 0.0589 & $\mathbf{0 . 9 5 1 6}$ & 0.0502 & 0.0657 & 0.0164 & 0.0415 & 0.0579 & $\mathbf{1 . 0 0 0 0}$ \\
\hline II & $\mathbf{0 . 9 9 9 0}$ & 0.2869 & 0.2067 & $\mathbf{0 . 9 2 7 5}$ & $\mathbf{0 . 9 9 9 0}$ & $\mathbf{0 . 9 9 7 1}$ & 0.4579 & $\mathbf{0 . 9 2 1 7}$ & $\mathbf{0 . 8 9 2 7}$ \\
\hline III & 0.0859 & $\mathbf{0 . 9 6 9 0}$ & $\mathbf{1 . 0 0 0 0}$ & 0.1632 & 0.0995 & 0.1391 & 0.2531 & $\mathbf{0 . 8 6 5 7}$ & $\mathbf{1 . 0 0 0 0}$ \\
\hline IV & 0.0000 & 0.0000 & 0.0483 & 0.0000 & 0.0541 & 0.1140 & 0.0309 & $\mathbf{0 . 7 9 4 2}$ & $\mathbf{0 . 6 3 8 6}$
\end{tabular}

Table 1. Statistical power (higher the better) of various methods for hypothesis testing with threshold 0.05. The power was computed with respect to empirical surrogate distribution. Data set I: a two action potential model with or without correlation, II: homogeneous Poisson with different rate, III: precisely timed spike train and Poisson equivalent, IV: renewal process with or without serial correlation. 40 spike trains are used for I, II, and III, 320 spike trains are used for IV. FR: absolute difference between mean firing rate, FF: Fano factor difference, ISI: KS-statistic of inter-spike interval distribution, N: KS-statistic of total spike count distribution, $L_{2} 10, L_{2} 100$ : mean square difference of smoothed PSTH with corresponding kernel size, TTFS: KS-statistic of time to first spike distribution HL10, HL100: proposed non-parametric method with $100 \mathrm{~ms}$ and $10 \mathrm{~ms}$ kernel size $\sigma_{1}$

10 active channels in a randomized order. For analysis a moving window of 40 consecutive spike train responses, corresponding to a single selected stimulation site, were used to estimate the model.

\subsection{Assumptions and Surrogate data}

We assume that the culture reaches a stationary state at the end (last 80 trials out of 600 (or 1200) trials over 5 (or 10) minutes), and we want to determine how much more one can extend the window that is within the stationarity condition. The null hypothesis is that the set of trials of interest came from the same point process as the last 40 trials.

We used a resampling technique to create the surrogate distribution of divergences for the null hypothesis. First recall that the last 80 trials are i.i.d, by the previous assumption. We randomly choose 40 of the last 80 trials to divide into two. The divergence between these two halves is repeatedly computed for 2000 times to create the empirical distribution under the null hypothesis. The actual divergence value is compared against this to test if it significantly deviates from the null hypothesis.

\subsection{Multi-Channel Recording}

To utilize the multi-channel recording nature of the experiments, we assumed that the channels have similar non-stationarity pattern over time. By averaging over the channels, a smoother estimate of the divergence is obtained. The distribution for null hypothesis must also be changed accordingly by taking the mean of across channels.

We have selected 5 to 15 active channels with phasic responses which seems to be changing in the experimental setup. We removed the channels that does not have less than or equal to 5 action potentials for $90 \%$ of the trials. The inactive channels that cannot be reliably estimated (mean number of action potentials less than 0.5 ) are removed from analysis as well. This process also reduces the bias and increases sensitivity in some cases. Including all the channels for analysis does not change the result significantly.

\subsection{Analysis of MEA data}

Figure 1 shows that the cultures are non-stationary for the first 350 samples corresponding to 3 minutes. The dashed line, and the dotted line correspond to empirical and gaussian assumed 95 per cent confidence level for the null hypothesis. The divergence values (blue and green) have decreasing trend during this period and cross the threshold. The non-stationarity (deviation from the null-hypothesis) is steep for the first 200 samples, followed by a plateau. These observations can also be made with other methods except ISI and FF.
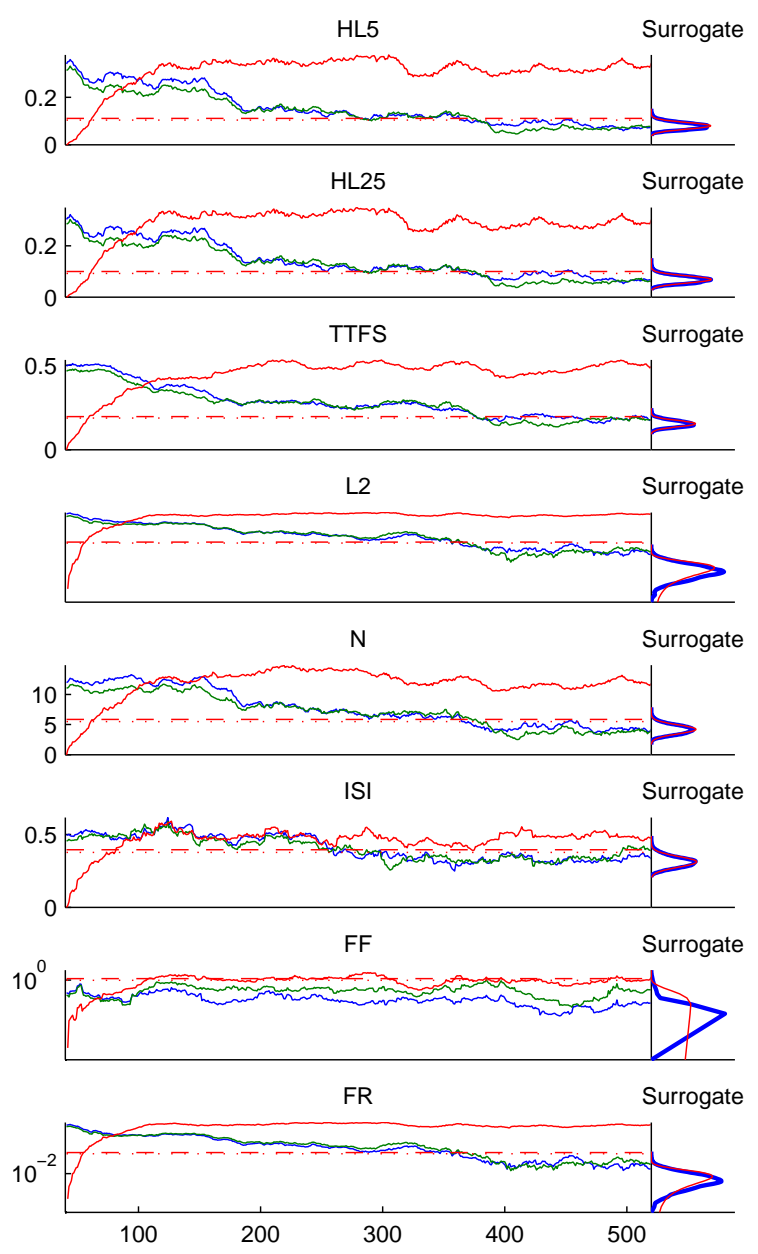

Fig. 1. X-axis is stimulation index corresponding to 20 stimulations a second (sample time). Y-axis are the divergence values for each method. The distribution of divergences under the null hypothesis (see detailed assumptions in the text) is shown in the right side (red curve is the estimated gaussian assumption). Each curve is the divergence between the sliding window of 40 samples. The red, blue and green curves are against the first 40 samples, last 40 samples, second last 40 samples, correspondingly. Note that the divergence reaches zero when the window coincides. The divergence values for FR, FF, and L2 are in log scale. The smoothing kernel size for L2, HL5, HL25 were $5 \mathrm{~ms}, 5 \mathrm{~ms}$, and $25 \mathrm{~ms}$, correspondingly. 


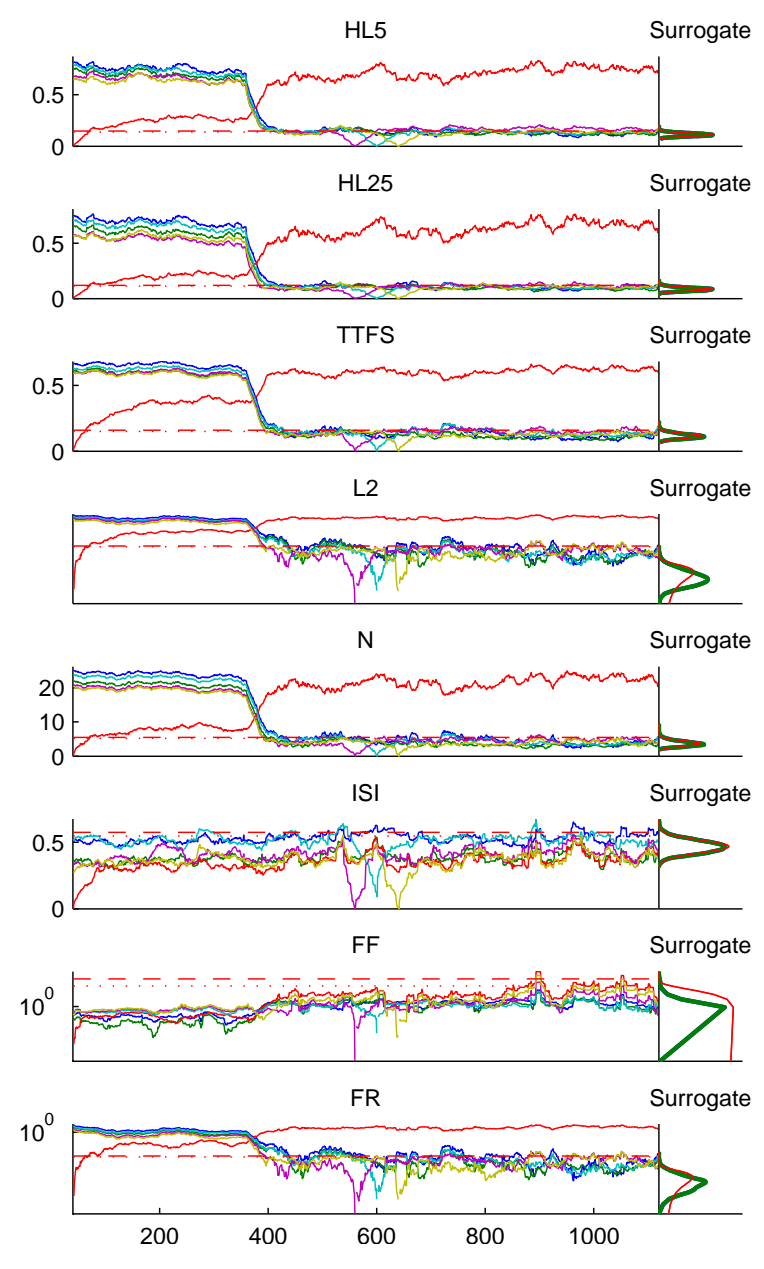

Fig. 2. Same as figure 1, but on a different culture with a lengthened protocol; two 5 minute stimulation sessions separated by 5 minutes at 600 sample time. Three additional reference time periods for divergence estimation are added as well; two just before and one after the resting period. No stimulation was applied during the 5 minute rest, and any short-term effect is presumed to be reversed.

Figure 2 shows a more discontinuous state change at around 400 samples. Despite a 5 minute rest at 600 samples time point, the culture response is still within the pseudo-stationary region. Hence, the sudden change is likely a long-term plasticity effect. Again similar observations can be made with different divergence measures. Depending on cultures, we could distinguish short-term changes and long-term changes $(N=6)$.

\section{DISCUSSION}

For the presented recorded data from cultures, the non-stationarity was both in the form of rate change, and spike timing change. The time to first spike method was most sensitive in detecting nonstationarity for both datasets presented, however, as demonstrated with the artificial data sets, it fails to detect it in general cases.

The smoothing kernel size $\sigma_{1}$ is a free parameter of the divergence measure. As can be seen from the results, the method is quite robust in terms of the choice of $\sigma_{1}$ when precise timing is not impor- tant. However, it should be noted that for smaller kernel size point processes will be considered all different, and for larger kernel size they will be considered all similar; either extreme would result in low sensitivity. For neural data, physiological time scale $1 \mathrm{~ms}$ to 100 ms would be a good starting point.

Due to its generality and good sensitivity, we can use the proposed method to claim pseudo-stationarity (non-stationarity not detected). As demonstrated, it is possible to use it for change point detection, and plasticity detection. Another possible application is to project spike train series into a dissimilarity feature space [13].

\section{REFERENCES}

[1] Karl Dockendorf, Il Park, Ping He, José C. Príncipe, and Thomas B. DeMarse, "Liquid state machines and cultured cortical networks: The separation property," Biosystems, vol. 95, no. 2, pp. 90-97, February 2009.

[2] D. J. Daley and D. Vere-Jones, An Introduction to the Theory of Point Processes, Springer, 1988.

[3] Tommi Jaakkola, Mark Diekhans, and David Haussler, "Using the fisher kernel method to detect remote protein homologies," in Proceedings of the Seventh International Conference on Intelligent Systems for Molecular Biology. 1999, pp. 149-158, AAAI Press.

[4] Tony Jebara, Risi Kondor, and Andrew Howard, "Probability product kernels," J. Mach. Learn. Res., vol. 5, pp. 819-844, 2004.

[5] Matthias Hein and Olivier Bousquet, "Hilbertian metrics and positive definite kernels on probability measures," in Proceedings of the Tenth International Workshop on Artificial Intelligence and Statistics, 2005, pp. 136-143.

[6] Jian Wu Xu, António R. C. Paiva, Il Park, and José C. Príncipe, "A reproducing kernel Hilbert space framework for information-theoretic learning," Signal Processing, IEEE Transactions on, vol. 56, no. 12, pp. 5891-5902, 2008.

[7] Conor Houghton, "Studying spike trains using a van Rossum metric with a synapse-like filter," Journal of Computational Neuroscience, vol. 26, no. 1, pp. 149-155, February 2009.

[8] António R. C. Paiva, Il Park, and José Príncipe, "A reproducing kernel Hilbert space framework for spike trains," Neural Computation, vol. 21, no. 2, pp. 424-449, Feburary 2009.

[9] Jean-Marc Fellous, Paul H. E. Tiesinga, Peter J. Thomas, and Terrence J. Sejnowski, "Discovering spike patterns in neuronal responses," J. Neurosci., vol. 24, no. 12, pp. 2989-3001, Mar. 2004.

[10] D. H. Hubel and T. N. Wiesel, "Receptive fields of single neurones in the cat's striate cortex.," The Journal of physiology, vol. 148, pp. 574-591, October 1959.

[11] Michael R. DeWeese, Michael Wehr, and Anthony M. Zador, "Binary spiking in auditory cortex," J. Neurosci., vol. 23, no. 21, pp. 7940-7949, Aug. 2003.

[12] Maurice J. Chacron, Benjamin Lindner, and André Longtin, "Noise shaping by interval correlations increases information transfer," Physical Review Letters, vol. 92, no. 8, pp. 080601+, Feb 2004.

[13] Elzbieta Pekalska and Robert P. W. Duin, The Dissimilarity Representation for Pattern Recognition, World Scientific Publishing Co., Inc., River Edge, NJ, USA, 2005. 